\title{
Pancreatic Anastomotic Leakage
}

National Cancer Institute

\section{Source}

National Cancer Institute. Pancreatic Anastomotic Leakage. NCI Thesaurus. Code C78518.

Leakage due to breakdown of a pancreatic anastomosis. 